\title{
Study on Risk Factors and Preventive Measures of Abdominal Incision Infection in Obstetrics and Gynecology
}

\author{
Xiaojun Liu ${ }^{1}$, Lili Xuan*, ${ }^{1}$ \\ ${ }^{1}$ China-Japan Union hospital of Jilin University, Changchun, Jilin, 130033 \\ * corresponding author
}

Keywords: Obstetrics and Gynecology; preventive measures; infection risk factors

\begin{abstract}
To analyze the risk factors and preventive measures of abdominal incision infection in obstetrics and gynecology, and to provide clinical guidance for reducing the incision infection rate and improving the quality of prognosis, a total of 319 obstetrics and gynecology patients undergoing abdominal surgery from May 2009 to May 2012 were selected and divided into two groups: infection group (21 cases) and non-infection group (298 cases). Clinical data were compared between the two groups. Analyze risk factors and discuss interventions. Data are analyzed using SPSS13.0. A total of 21 cases of incision infection occurred in 319 patients. The infection rate was $6.58 \%$. Multivariate regression analysis revealed that age $\geq 60$ years, BMI $<19$ or $>23 \mathrm{~kg} / \mathrm{m} 2$, operative time $\geq 120 \mathrm{~min}$, ASA grade III grade, emergency surgery. Noninvasive physicians sutured, prophylactic use of antibiotics and no postoperative rehabilitation education were independent risk factors affecting abdominal surgical incision infection in obstetrics and gynecology $(\mathrm{P}<0.05)$. Obstetric abdominal surgery incision infection is closely related to the patient's own factors and surgical factors. It is necessary to pay attention to correct the poor state of the patient's immune function, and strictly implement the principle of asepsis to reduce the incidence of incision infection, improve patient prognosis and survival quality.
\end{abstract}

\section{Introduction}

Incision infection is one of the common complications of abdominal surgery for obstetrics and gynecology. It accounts for $43.49 \%$ of postoperative infections. It affects the incision healing to a lesser extent, and it can cause uterine cavity infections, late postpartum hemorrhage, sepsis, and even endanger the lives of women. Lead to medical disputes and have adverse effects on quality of life and prognosis. Through the investigation and analysis of the abdominal surgery in obstetrics and gynaecology in our hospital, the risk factors and prevention measures of incision infection were discussed.

\section{Materials and Methods}

A total of 319 obstetrics and gynecology patients undergoing abdominal surgery were selected

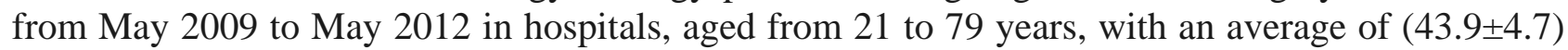
years; the disease duration ranged from 2 months to 5 years. At an average of $(1.7 \pm 0.4)$ years, 21 patients were infected and 298 patients were non-infected.

Referring to the "Diagnosis Criteria for Nosocomial Infections" issued by the Ministry of Health [3], the incision infection occurred within one month after surgery was judged: there were red, swollen, hot, and painful infection symptoms, and purulent secretion occurred at the superficial incision. The substances and secretions are positive for pathogens and the site of infection may include skin or subcutaneous tissue at the incision.

Patients with confirmed incision infections were included in the infection group and other patients were included in the non-infected group. Clinical data such as age, body mass index (BMI), operating time, classification of the American Anesthesiologists (ASA), and type of surgery were compared between the two groups. [4], Incorporating statistically significant factors into logistic 
multivariate regression analysis. For the risk factors affecting abdominal incision infection in obstetrics and gynecology, refer to clinical experience and relevant literature, and propose corresponding countermeasures. All data from this clinical study were analyzed using SPSS 13.0. Chi-square test was used for counting data and t-test was used for measurement data. Logistic multivariate regression analysis was performed to determine risk factors affecting abdominal incision infection in obstetrics and gynecology. For $\mathrm{a}=0.05, \mathrm{P}<0.05$ for the difference was statistically significant.

Nineteen cases of incision infection occurred in 19 patients and the infection rate was 6.58\%. The differences in age, BMI, operative time, ASA grade, type of surgery, prophylactic antibiotic use, and postoperative rehabilitation education were statistically significant $(\mathrm{P}<0.05)$. Multivariate regression analysis found that age $\geq 60$ years, $\mathrm{BMI}<19$ or $>23 \mathrm{~kg} / \mathrm{m} 2$, operative time $\geq 120 \mathrm{~min}$, ASA grade III grade, emergency surgery, non-head surgeon suture, prophylactic use of antibiotics and no postoperative rehabilitation Education is an independent risk factor that influences abdominal wound incision in obstetrics and gynecology $(\mathrm{P}<0.05)$.

\section{Discussion}

With the improvement of medical level and anesthesia technology, the healing of abdominal incisions in obstetrics and gynecology has been well guaranteed, but some patients still have complications such as incision infection, dehiscence, or non-healing, which brings about the physical and mental health of patients. The adverse effects have increased the economic burden on patients. Therefore, effective prevention of abdominal incision infections in obstetrics and gynecology is the key to improving patient prognosis and relieving the relationship between doctors and patients. In the survey of 319 patients, there were 21 cases of incision infection in the Chinese Communist Party, and the infection rate was $6.58 \%$. This is similar to the literature [5], showing that incision infection is still the most common complication after abdominal surgery in the obstetrics and gynecology department. Patients were grouped according to their infections. Multivariate logistic regression analysis revealed that patients aged $\geq 60$ years, BMI $<19$ or $>23$ $\mathrm{kg} / \mathrm{m} 2$, operative time $\geq 120 \mathrm{~min}$, ASA grade III, emergency surgery, and non-head surgeon Prophylactic use of antibiotics and the lack of postoperative rehabilitation education are independent risk factors affecting abdominal incision infections in obstetrics and gynecology. The main causes are: (1) Older patients often have coronary heart disease, hypertension, diabetes, etc. A variety of basic diseases, especially female patients, are more likely to be accompanied by symptoms such as anemia and hypoproteinemia, which leads to poor autoimmune function, low immune defense capability, and slower wound healing, which is vulnerable to pathogenic bacteria. (2) BMI $<19$ or $>23 \mathrm{~kg} / \mathrm{m} 2$ indicates malnutrition or obese patients. Malnutrition patients need longer recovery time, and their hospitalization time is also prolonged, which increases the time of exposure to pathogenic bacteria in the hospital and increases the risk of infection. However, in obese patients, it was confirmed that the blood volume was significantly lower than that of normal BMI patients, and their tissue insufficiency resulted in a decrease in wound healing time and resistance. (3) Increased surgical time leads to an increase in the amount of blood loss in the patient, and it is more likely that events such as poor postoperative incision cleanliness increase the risk of infection. (4) Extensive emergency surgery The current situation of short skin preparation time and inadequate medical device disinfection have made it impossible to fully implement the aseptic principle in the procedure, which has led to a significant increase in the risk of pathogen infection of wounds. (5) ASA grade III patients have a severe disease condition with a high risk of anesthesia. During the surgery, they are prone to complications. Functional compensation is affected after surgery and the ability to resist is decreased, making the ability to prevent the commensal bacteria from being insufficient. Most scholars hold different opinions on whether or not to use antibiotics prophylactically after surgery. Postoperative use of antibacterial drugs can reduce the incidence of infection in patients, but the authors found that the majority of patients in this survey were treated with prophylactic antibiotics, but the prevention effect was poor, and even increased the risk of infection, so in the future in clinical treatment, the application time and drug selection of 
antimicrobial drugs should be controlled to ensure rational use of drugs.

\section{Infection Factors and Countermeasures}

(1) Incision infection caused by obesity: One of the main factors of obesity and incision infection, obese patients have low blood volume, weak antibodies, and the operation time is longer for those who are relatively thin, prolong the postoperative recovery time, and easily lead to infection; Therefore, the medical staff should guide the obese patients to reasonably control their weight, avoid the thick fat layer in obese patients, affect the operative field and operation, and guide the patients to eat reasonably and eat high-fat foods before the surgery. Eating fruits and vegetables can not only control body weight, but also play a role in the healing of postoperative incisions. (2) Incision infection caused by long operation time: The longer the operation time, the greater the amount of bleeding and the greater the chance of infection; Therefore, to improve the surgeon's professional skills and surgical level, can effectively reduce the incision infection; (3) incision infection caused by incomplete disinfection: disinfection of patients is not in place, will also increase the chance of wound infection, which can be avoided Factors, as long as the medical staff are dedicated, careful, and careful, can avoid the wound infection caused by this aspect; (4) Preventive medication Notch improper infection: rational drug medical staff should avoid the use of broad-spectrum antimicrobial drugs; (5) the underlying cause wound infection disease: diabetes, bronchitis and other underlying disease, also wound infection. Diabetic patients suffer from a certain degree of destruction of the immune system, the regeneration ability is not strong, the ability to resist and repair is not good, the incision is susceptible to infection, poor healing ability; bronchitis or a cold causes postoperative cough, but also induces increased abdominal incision tension, increased abdominal pressure, thereby affecting incision healing. Actively prevent and treat primary diseases. Therefore, patients with colds should take therapeutic drugs with good efficacy and effective treatment; patients with bronchitis should choose to reduce the treatment of cough and prevent them from taking drugs; patients with diabetes should adjust their blood glucose levels before surgery and wait for fasting blood glucose to be below $8 \mathrm{mmol} / \mathrm{L}$. Surgery; patients with edema, the edema is controlled under mild conditions and then proceed to surgery; patients with anemia, hemoglobin increased to $90 \mathrm{~g} / \mathrm{L}$ or more before surgery; (6) surgical instruments and incision and suture technology is also affecting incision healing the elements of.

In recent years, high-frequency electric knife is widely used in clinical practice, increasing the chance of incision infection. In addition, incision and suturing technology is also a factor that can affect the wound healing can not be ignored: repeated cutting of adipose tissue, suture too tight, too dense, too loose, leak seam, leaving a dead space, incision time is too long and so on. Therefore, doctors should be serious, careful, and professional when performing surgery on patients. When doing an abdominal wall incision, try to shorten the contact time between the electrocautery and the adipose tissue, and cut the subcutaneous fat layer as much as possible to avoid leaving the dead space due to excessive thickness of the fatty layer when repeatedly cutting the fat layer and suturing the wound. Protect the fat layer with saline gauze, stop the bleeding completely, and suture properly to avoid dislocation. Surgery to minimize incision exposure time. Other incision infections, such as ward contamination, premature dressing changes, and age, accounted for a relatively small proportion. The relevant factors analyzed in the present study were basically consistent with the existing reports, of which obesity, antibacterial use, and disinfection accounted for a high proportion of infections. It is of great significance for clinicians to understand various factors related to incision infection to prevent the occurrence of infection.

\section{Conclusion}

With the continuous improvement of medical technology, the healing effect of abdomen surgical incisions of obstetrics and gynecology has been continuously improved, but the incision infection rate remains high, which seriously affects the patient's physical and mental health. It is of great significance for doctors and nurses to understand various factors related to incision infection in the 
prevention of infection. Focusing on factors affecting incision fat liquefaction, reasonable and effective prevention, treatment and nursing measures can not only reduce the incidence of incision infection, avoid the occurrence of doctor-patient accidents, but also accelerate the incision healing of patients and promote the patients to recover as soon as possible.

\section{References}

[1] Zhou Haiyan. Obstetrics and Gynecology Abdominal incision intradermal suture 186 cases of observation and analysis [J]. China Health Monthly, Theory, 2011 (8): 114.

[2] Gao Yuanyuan. Analysis of related factors of abdominal incision infection in obstetrics and gynecology[J]. China Practical Medicine,2012,21(7):119-121.

[3] Xun Xunhong. The cause of abdominal incision infection in obstetrics and gynecology and its prevention and control methods[J]. Chinese Health Industry, 2011, 8(29): 84.

[4] Yan Xiaocui, Zhou Maoying, Zhang Tengfei. Investigation and analysis of risk factors for abdominal incision infection in obstetrics and gynecology[J]. Chinese Journal of Nosocomiology, 2010,20(7):935-936.

[5] Ye X, Zheng XH, Ge C L. Causes and prevention of abdominal incision infection in obstetrics and gynecology[J]. Jilin Medical Journal,2009,30(18):2119-2120. 